\title{
REFINEMENTS ON THE INTERLACING OF EIGENVALUES OF CERTAIN TOTALLY NONNEGATIVE MATRICES
}

\author{
Shaun M. Fallat ${ }^{1}$ AND Hugo J. WoERdeman ${ }^{2}$
}

\begin{abstract}
It has long been known that the eigenvalues of a totally positive matrix interlace the eigenvalues of its maximal leading principal submatrix. Motivated by recent questions arising from studying the roots of certain biorthogonal polynomials, we extend the classical strict interlacing fact to other classes of totally nonnegative matrices.
\end{abstract}

Mathematics subject classification (2000): 15A48, 15A13, 33C45.

Key words and phrases: Totally nonnegative matrices, oscillatory matrices, eigenvalues, eigenvalue interlacing, biorthogonal polynomials.

\section{REFERENCES}

[1] T. ANDO, Totally Positive Matrices, Linear Algebra Appl., 90 (1987) 165-219.

[2] M. Bertola, B. EYNARD, AND J. HARNAD, Duality, biorthogonal polynomials and multi-matrix models, Comm. Math. Phys., 229, no. 1, (2002) 73-120.

[3] T. S. CHIHARA, An introduction to orthogonal polynomials, Mathematics and its Applications, Vol. 13, Gordon and Breach Science Publishers, New York-London-Paris, 1978.

[4] C. W. CRYer, Some Properties of Totally Positive Matrices, Linear Algebra Appl., 15 (1976) 1-25.

[5] C. DE BOOR AND A. PINKUS, The approximation of a totally positive band matrix by a strictly banded totally positive one, Linear Algebra Appl., 42 (1982) 81-98.

[6] N. M. ERCOLANI AND K. T.-R. MCLAUGHLIN, Asymptotics and integrable structures for biorthogonal polynomials associated to a random two-matrix model, Phys. D, 152/153 (2001) 232-268.

[7] S. M. FALLAT, Bidiagonal Factorizations of Totally Nonnegative Matrices, American Math. Monthly 109 (2001) 697-712.

[8] S. M. Fallat, A Remark on Oscillatory Matrices, Linear Algebra Appl., 393 (204) 139-147.

[9] S. M. Fallat, M. I. GeKhtman, AND C. R. Johnson, Spectral structures of irreducible totally nonnegative matrices, SIAM J. Matrix Anal. Appl., 22 (2000) 627-645.

[10] S. Fomin AND A. Zelevinsky, Total positivity: Tests and parameterizations, Math. Intelligencer, 22 (2000) 23-33.

[11] F. R. Gantmacher And M. G. Krein, Oscillation Matrices and Kernels and Small Vibrations of Mechanical Systems, AMS, Providence, 2002. Oszillationsmatrizen, Oszillationskerne und kleine Schwingungen Mechanischer Systeme, Akademie-Verlag, Berlin, 1960.

[12] M. GasCA AND C. A. MicChElli, Total Positivity and its Applications, Mathematics and its Applications, Vol. 359, Kluwer Academic, Dordrecht, 1996.

[13] R. A. Horn AND C. R. Johnson, Matrix Analysis, Cambridge University Press, New York, 1985.

[14] S. KaRlin, Total Positivity, Vol. I, Stanford University Press, Stanford, 1968.

[15] S. KARLIN AND A. PINKUS, Oscillation properties of generalized characteristic polynomials for totally positive and positive definite matrices, Linear Algebra and Appl., 8 (1974) 281-312.

[16] A. B. J. KUIJLAARS AND K. T.-R. MCLAUGHLin, A Riemann-Hilbert problem for biorthogonal polynomials, J. Comput. Appl. Math. 178 (2005) 313-320.

[17] C.-K. LI AND R. MATHIAS, Interlacing inequalities for totally nonnegative matrices, Linear Algebra Appl., 341 (2002) 35-44. 
[18] A. PINKUS, An Interlacing Property of Eigenvalues of Strictly Totally Positive Matrices, Linear Algebra Appl., 279 (1998) 201-206.

[19] J. W. RAINEY AND G. J. HALBeTLER, Tridiagonalization of Completely Nonnegative Matrices, Math. Comp., 26 (1972) 121-128.

[20] H. J. WOERDEMAN, Interlacing properties or roots of certain biorthogonal polynomials, J. Approximation Theory 143 (2006) 150-158.

[21] A. Whitney, A Reduction Theorem for Totally Positive Matrices, J. Analyse Math., 2 (1952) 88-92. 